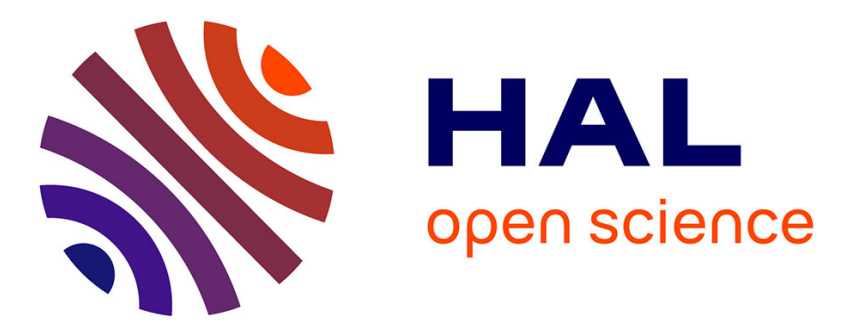

\title{
Etude théorique d'un dispositif permettant de condenser un faisceau laser gaussien en une tache carrée de dimension variable avec une répartition d'énergie homogène
}

\author{
Li Junchang, J. Merlin, T. Manderscheid, A. Corbet
}

\section{To cite this version:}

Li Junchang, J. Merlin, T. Manderscheid, A. Corbet. Etude théorique d'un dispositif permettant de condenser un faisceau laser gaussien en une tache carrée de dimension variable avec une répartition d'énergie homogène. Revue de Physique Appliquée, 1989, 24 (12), pp.1111-1118. 10.1051/rphysap:0198900240120111100 . jpa-00246148

\author{
HAL Id: jpa-00246148 \\ https://hal.science/jpa-00246148
}

Submitted on 1 Jan 1989

HAL is a multi-disciplinary open access archive for the deposit and dissemination of scientific research documents, whether they are published or not. The documents may come from teaching and research institutions in France or abroad, or from public or private research centers.
L'archive ouverte pluridisciplinaire HAL, est destinée au dépôt et à la diffusion de documents scientifiques de niveau recherche, publiés ou non, émanant des établissements d'enseignement et de recherche français ou étrangers, des laboratoires publics ou privés. 


\title{
Etude théorique d'un dispositif permettant de condenser un faisceau laser gaussien en une tache carrée de dimension variable avec une répartition d'énergie homogène
}

\author{
Li Junchang (*), J. Merlin, T. Manderscheid (**) et A. Corbet (**) \\ INSA Lyon, Bât. 502, G.E.M.P.P.M. (U.A. 341) Calfetmat, 69621 Villeurbanne Cedex, France
}

(Reçu le 17 avril 1989, révisé et accepté le 7 septembre 1989)

\begin{abstract}
Résumé. - Partant d'un faisceau laser primaire gaussien nous proposons un dispositif permettant d'obtenir par formation d'images des répartitions énergétiques homogènes sur des sections carrées de dimensions variables. Ce dispositif est composé de 2 miroirs prismatiques à 4 faces, de 2 miroirs plans et d'une lentille. Son étude théorique complète a été réalisée pour uñ positionnement idéal des différents éléments le constituant. Les influences d'écarts à ces positions idéales ont également été étudiées afin de définir les tolérances à respecter pour la réalisation et l'utilisation du dispositif. Sa compacité et sa simplicité de conception doivent permettre de l'utiliser pour la réalisation industrielle de traitements thermiques superficiels.
\end{abstract}

\begin{abstract}
A device able to transform a primary Gaussian laser beam into square homogeneous repartition with variable sizes is suggested. This device consist of prismatical mirrors with 4 sides, of 2 plane mirrors and of a lens. Whole theoretical analysis has been performed with different optical elements located in ideal positions and with gaps to these positions to estimate mechanical allowances necessary to achieve and employ this device. Its compacity and simplicity may be able to use it for industrial thermal treatments with laser beam.
\end{abstract}

\section{Introduction.}

Dans le but de l'utilisation de sources laser pour la réalisation de traitements superficiels homogènes sur des matériaux, nous avons déjà étudié un dispositif permettant de transformer un faisceau laser gaussien $\left(\mathrm{TEM}_{00}\right)$ en une répartition uniforme [1]. Nous avons également montré qu'en ajoutant une lentille sur le parcours du faisceau incident, il est possible de diminuer le grandissement global du système optique et par suite d'obtenir une tache «laser » avec une répartition homogène de grande densité d'énergie [2]. Mais il restait un gros problème à résoudre au niveau de l'élément optique principal du dispositif qui devait obligatoirement recevoir une grande densité d'énergie, ce qui limitait l'intérêt du dispositif. Nous avons donc poursuivi cette étude sur le plan théorique, toujours sur la base de la superposition des images des champs de quatre quarts du faisceau principal, mais en nous attachant à obtenir

$\left(^{*}\right)$ Adresse permanente: Institut de technologie de Kunming Yunnan, République Populaire de Chine.

$(* *)$ Société PSA, Centre technique Citroën, 78140 Vélizy, France. un dispositif d'une conception plus simple que les précédents et surtout plus susceptible de supporter de grandes densités d'énergie.

\section{Présentation générale du dispositif.}

Ce dispositif est schématisé sur les figures $1 \mathrm{a}$ et $1 \mathrm{~b}$, il est composé de quatre éléments optiques. Le faisceau laser primaire incident de longueur d'onde $\lambda$ et de mode $\mathrm{TEM}_{00}$ avec un rayon $w$ à $1 / e^{2}$ est tout d'abord divisé et réfléchi par le miroir $M_{1}$ constitué de quatre faces réfléchissantes. Il en résulte quatre sous-faisceaux symétriques par rapport au centre du faisceau primaire. Ces sous-faisceaux, après deux réflexions par deux miroirs plans désignés $M_{2}$ et $M_{3}$, sont à nouveau réfléchis par le miroir $\mathbf{M}_{4}$ analogue au miroir $\mathbf{M}_{1}$, et ils sont symétriques selon un plan perpendiculaire à l'axe du faisceau primaire. Il en résulte une inclinaison $\phi$ des quatre sous-faisceaux par rapport à la direction du faisceau primaire ; sous-faisceaux qui traversent ensuite la lentille $\mathrm{L}_{\mathrm{t}}$. En considérant les quatre plans-objets comme les plans passant par le sommet du miroir $\mathbf{M}_{1}$ et faisant un angle de $\pi / 2-\phi$ par rapport aux directions des 
faisceaux réfléchis, il est évident qu'on peut trouver un plan de superposition, sur lequel il y a une tache carrée virtuelle formée par la superposition des 4 images virtuelles des quatre quarts de faisceau, situées à une distance $d_{0}$ de la lentille $\mathrm{L}_{\mathrm{t}}$. Ainsi, après avoir traversé ce système optique, on pourra obtenir une image réelle ayant une forme carrée et située à une distance $d_{\mathrm{i}}$ sous cette lentille. Partant d'un faisceau laser idéal gaussien il est facile de montrer que la répartition d'énergie de la tache recomposée dans le plan-image sera sensiblement uniforme à partir du moment où la structure du dispositif conduira à une tache carrée virtuelle dont le côté $2 L$ après la recomposition sera environ égal à $1,1 w[1]$.

Evidemment, la dimension de l'image réelle sera fonction du choix de la distance focale de la lentille. Il doit ainsi être possible d'obtenir des taches laser carrées de dimensions variables avec une répartition d'énergie uniforme à partir du même système optique par utilisation de différentes lentilles. Cela devrait permettre de répondre à un certain nombre de problèmes posés par les traitements superficiels des matériaux. Les précédents dispositifs proposés ayant été désignés $L_{1}, L_{2}$ [1] nous désignerons celuici $\mathrm{L}_{3}$.

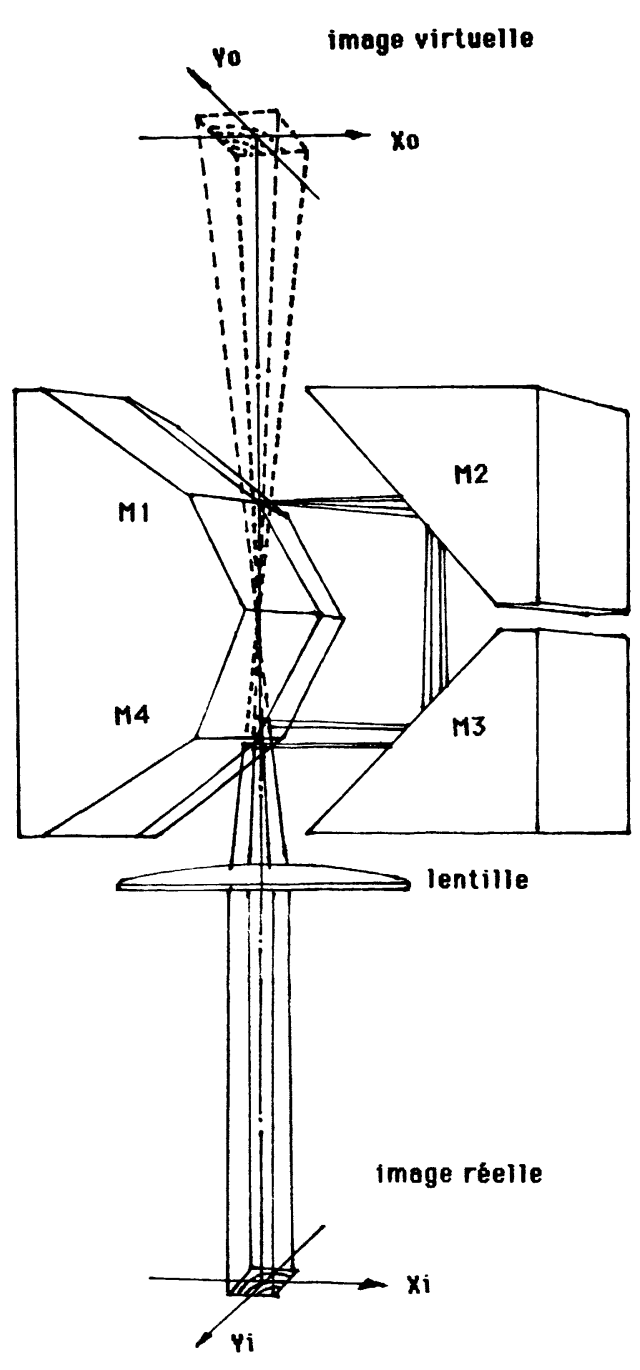

a)

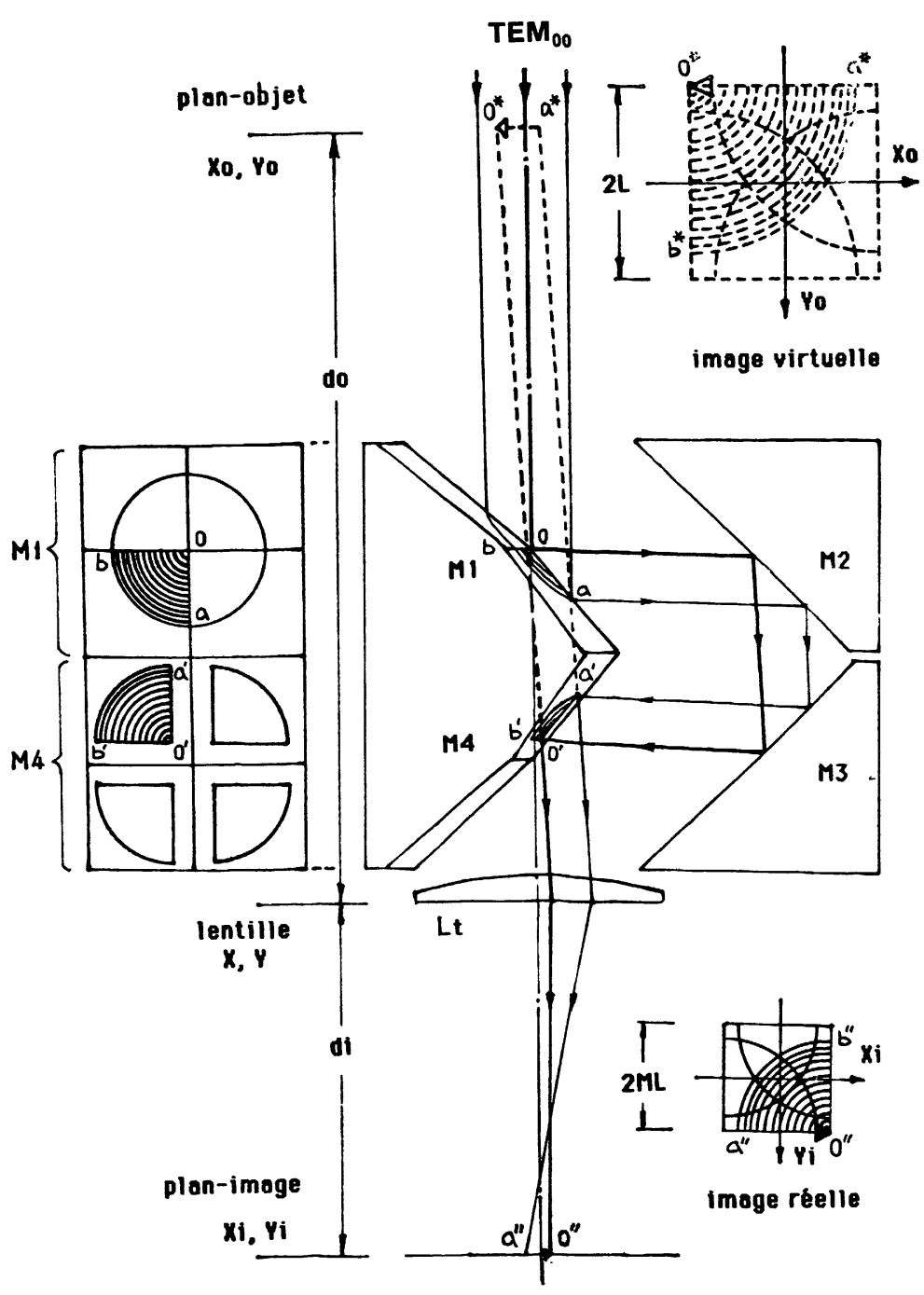

b)

Fig. 1. - Dispositif de recombinaison de faisceaux (« $\left.\mathrm{L}_{3} »\right)$ : a) vue en perspective ; b) vue en projection, schématisant la trajectoire d'un sous-faisceau.

[ $\mathrm{L} 3$ » device : a) perspective view ; b) projection view schematizing a « sub-beam » trajectory.] 


\section{Analyse de la répartition énergétique après recom- position.}

Par raison de symétrie géométrique. Il suffit de calculer une image d'un des quatre sous-faisceaux pour accéder à la répartition d'énergie totale. Sur la figure 1 sont mentionnés les chemins optiques du dispositif équivalent ainsi que le repérage des axes de coordonnées. Afin de simplifier les calculs les approximations suivantes sont effectuées :

a) on a supposé que la divergence du faisceau était nulle, bien que cela ne soit pas exact, mais des calculs plus détaillés permettent de montrer qu'une faible divergence ne modifie pas de manière notable les résultats obtenus en considérant un faisceau rigoureusement parallèle ;

b) l'angle d'incidence des quatre sous-faisceaux sur la lentille étant petit, l'amplitude complexe d'une image virtuelle (Fig. 1) peut être approximée par :

$$
\begin{array}{r}
U_{0}\left(X_{0}, Y_{0}\right)=\exp \left[\frac{\left(X_{0}+L\right)^{2}+\left(Y_{0}+L\right)^{2}}{W^{2}}\right] \times \\
\times \exp \left[j k S\left(X_{0}+Y_{0}\right)\right]
\end{array}
$$

avec

$2 L=1,1 W ; k=2 \pi / \lambda, \lambda$ longueur d'onde ;

$S=(\sqrt{2} / 2) \sin \phi$;

$-L \leqslant X_{0} \leqslant 2 W-L, \quad-L \leqslant Y_{0} \leqslant 2 W-L$.

c) la lentille est supposée de forme carrée de côté $2 a$; la pupille de sortie peut alors être considérée comme ayant une transmittance en amplitude donnée par :

$$
P(X, Y)= \begin{cases}1 & \text { si } 0 \leqslant X \leqslant a, \quad 0 \leqslant Y \leqslant a \\ 0 & \text { ailleurs }\end{cases}
$$

d) les temps d'interaction rayonnement-matériaux sont tels que les effets thermiques induits dans les matériaux peuvent généralement être considérés comme fonction de l'énergie moyenne incidente sur une zone de quelques centaines de $\mu \mathrm{m}$. La périodicité de la structure interférentielle résuitant des interférences des quatre sous-faisceaux ayant cet ordre de grandeur [3], nous la négligerons. La répartition d'énergie finale pourra être considérée comme étant la somme des intensités des quatre sous-faisceaux.

A partir de ces approximations et selon la théorie de l'optique de Fourier, l'amplitude complexe du champ dans le plan-image s'exprime par le produit de convolution de la réponse impulsionnelle de la pupille de sortie avec l'image de l'optique géométrique [4] :

$$
U_{\mathrm{i}}\left(X_{\mathrm{i}}, Y_{\mathrm{i}}\right)=h\left(X_{\mathrm{i}}, Y_{\mathrm{i}}\right) \otimes \frac{1}{M} U_{0}\left(-\frac{X_{\mathrm{i}}}{M},-\frac{Y_{\mathrm{i}}}{M}\right)
$$

où

$M=d_{1} / d_{0}$, grandissement transversal de l'image ;

$$
\begin{aligned}
h\left(X_{\mathrm{i}}, Y_{\mathrm{i}}\right)=\iint_{-\infty}^{\infty} P\left(\lambda d_{\mathrm{i}} X, \lambda d_{\mathrm{i}} Y\right) \times \\
\quad \times \exp \left[-j 2 \pi\left(X X_{\mathrm{i}}+Y Y_{\mathrm{i}}\right)\right] \mathrm{d} X \mathrm{~d} Y .
\end{aligned}
$$

En évaluant $P\left(\lambda d_{\mathrm{i}} X, \lambda d_{\mathrm{i}} Y\right)$ dans cette expression, on peut obtenir :

$$
\begin{array}{r}
h\left(X_{\mathrm{i}}, Y_{\mathrm{i}}\right)=\left[\frac{\sin \left(\frac{\pi}{2 T} X_{\mathrm{i}}\right) \sin \left(\frac{\pi}{2 T} Y_{\mathrm{i}}\right)}{\pi^{2} X_{\mathrm{i}} Y_{\mathrm{i}}}\right] \times \\
\times \exp \left[-j \frac{\pi}{2 T}\left(X_{\mathrm{i}}+Y_{\mathrm{i}}\right)\right]
\end{array}
$$

avec

$$
T=\lambda d_{\mathrm{i}} / 2 a
$$

Ainsi, si l'on laisse de côté les déphasages qui n'interviennent pas dans l'évaluation de la répartition d'énergie, l'expression (3) peut se récrire :

$$
U_{\mathrm{i}}\left(X_{\mathrm{i}}, Y_{\mathrm{i}}\right)=\frac{1}{M} U_{\mathrm{i}}\left(X_{\mathrm{i}}\right) \cdot U_{\mathrm{i}}\left(Y_{\mathrm{i}}\right)
$$

avec

$$
\begin{aligned}
U_{\mathrm{i}}\left(X_{\mathrm{i}}\right)=\int_{-}^{M}(2 W-L) & {\left[\frac{\sin \frac{\pi}{2 T}\left(X_{\mathrm{i}}-X\right)}{\pi\left(X_{\mathrm{i}}-X\right)}\right] \times } \\
& \times \exp \left[-\frac{(X-M L)^{2}}{M^{2} W^{2}}\right] \\
& \times \exp \left[-j \frac{k}{M}\left(S-\frac{a}{2 d_{0}}\right) X\right] \mathrm{d} X \\
U_{\mathrm{i}}\left(Y_{\mathrm{i}}\right)=\int_{-}^{M L} & \\
& \times \exp \left[-\frac{(Y-L)\left[\frac{\sin \frac{\pi}{2 T}\left(Y_{\mathrm{i}}-Y\right)}{\pi\left(Y_{\mathrm{i}}-Y\right)}\right] \times}{M^{2} W^{2}}\right] \\
& \times \exp \left[-j \frac{k}{M}\left(S-\frac{a}{2 d_{0}}\right) Y\right] \mathrm{d} Y
\end{aligned}
$$

La densité d'énergie $E_{\mathrm{i}}\left(X_{\mathrm{i}}, Y_{\mathrm{i}}\right)$ au niveau de l'image d'un sous-faisceau est alors :

$E_{\mathrm{i}}\left(X_{\mathrm{i}}, Y_{\mathrm{i}}\right)=\frac{1}{M^{2}} U_{\mathrm{i}}\left(X_{\mathrm{i}}\right) \cdot U_{\mathrm{i}}^{*}\left(X_{\mathrm{i}}\right) \cdot U_{\mathrm{i}}\left(Y_{\mathrm{i}}\right) \cdot U_{\mathrm{i}}^{*}\left(Y_{\mathrm{i}}\right)$

et par raison de symétrie géométrique, la densité d'énergie totale $E$ après superposition des 4 sousfaisceaux est :

$$
\begin{aligned}
E=E_{\mathrm{i}}\left(X_{\mathrm{i}}, Y_{\mathrm{i}}\right)+E_{\mathrm{i}}( & \left.-X_{\mathrm{i}},-Y_{\mathrm{i}}\right)+ \\
& +E_{\mathrm{i}}\left(-X_{\mathrm{i}}, Y_{\mathrm{i}}\right)+E_{\mathrm{i}}\left(X_{\mathrm{i}},-Y_{\mathrm{i}}\right)
\end{aligned}
$$


Il est ainsi possible de déterminer la répartition énergétique au niveau de la zone de superposition; toutefois il n'est pas possible d'exprimer ce résultat au moyen de fonctions analytiques simples et seulement des résultats graphiques obtenus par calcul numérique seront fournis dans le paragraphe suivant.

\section{Analyse des expressions et simulation numérique.}

D'après les expressions précédentes, et à partir du moment où seront connus : le rayon $w$ du faisceau laser incident, sa longueur d'onde $\lambda$ et la dimension de la tache recomposée (grandissement $M$ ), il nous sera possible par calcul numérique d'obtenir la répartition théorique d'énergie dans la tache. Mais pour bien comprendre le résultat, il nous paraît intéressant, avant ce calcul, d'analyser plus en détail les deux expressions (6), (7).

De ces expressions il ressort que le facteur de phase complexe va immédiatement influencer le résultat du calcul numérique, ce facteur est en effet fonction des paramètres décrivant le dispositif ainsi que du déphasage du champ dans le plan-objet. Par conséquent, la qualité de l'image dépend non seulement des caractéristiques géométriques du dispositif, mais encore de la direction de propagation des sousfaisceaux incidents. Ainsi en choisissant judicieusement l'angle d'incidence $\phi$, ainsi que les deux paramètres $a$ et $d_{0}$, il sera possible d'améliorer la qualité de l'image par une conception optimale du système optique.

Pour analyser simplement les résultats des calculs numériques nous nous sommes donc d'abord placés dans le cas où la condition :

$$
S-\frac{a}{2 d_{0}}=0
$$

est satisfaite, ce qui d'après l'expression (2), correspond à :

$$
\sin \phi=a /\left(\sqrt{2} d_{0}\right)
$$

Dans cette situation, les facteurs de phase disparaissent et les deux expressions (6), (7) deviennent :

$$
\begin{aligned}
U_{\mathrm{i}}\left(X_{\mathrm{i}}\right)=\int_{-M(2 W-L)}^{M L} & \frac{\sin \frac{\pi}{2 T}\left(X_{\mathrm{i}}-X\right)}{\pi\left(X_{\mathrm{i}}-X\right)} \times \\
& \times \exp \left[-\frac{(X-M L)^{2}}{M^{2} W^{2}}\right] \mathrm{d} X \\
U_{\mathrm{i}}\left(Y_{\mathrm{i}}\right)=\int_{-M(2 W-L)}^{M L} & \frac{\sin \frac{\pi}{2 T}\left(Y_{\mathrm{i}}-Y\right)}{\pi\left(Y_{\mathrm{i}}-Y\right)} \times \\
& \times \exp \left[-\frac{(X-M L)^{2}}{M^{2} W^{2}}\right] \mathrm{d} Y
\end{aligned}
$$

Après comparaison avec les expressions obtenues pour le dispositif « $L_{1}$ » de la référence [1], on peut considérer que pour les deux dispositifs on obtiendra la même forme de répartition d'énergie dans les taches. Autrement dit, lorsque la condition (9) est satisfaite il suffira de remplacer la période spatiale $T$ de " $L_{1}$ " par $2 T$ pour que tous les résultats numériques du dispositif « $\mathrm{L}_{1}$ » soient transposables au présent dispositif « $\mathrm{L}_{3} »$. Toutefois, il faut noter que " $\mathrm{L}_{3}$ " pourra condenser un faisceau laser gaussien en une tache carrée de dimensions variables avec une répartition d'énergie homogène, ce qui n'était pas le cas pour « $\mathrm{L}_{1} »$.

Compte tenu de l'équipement à notre disposition (source CILAS CI 4000) nous nous sommes placés dans le cas d'une source laser $\mathrm{CO}_{2}$ continue $(\lambda=10,6 \mu \mathrm{m})$ ayant un rayon de faisceau $w$ de $7 \mathrm{~mm}$ et nous avons évalué la répartition énergétique dans le plan de superposition des images pour trois valeurs de grandissement transversal $M$, à savoir 0,5 1 et 1,5 . C'est-à-dire de manière à avoir après recomposition des taches carrées de $3,85 \mathrm{~mm}$ $7,7 \mathrm{~mm}-11,55 \mathrm{~mm}$ de côté (soit $2 M L=1,1 \mathrm{Mw}$ ). Ce qui correspond à des densités de puissance maximales comprises entre $2 \times 10^{4}$ et $2 \times 10^{3} \mathrm{~W} / \mathrm{cm}^{2}$ dans le cas d'une puissance transportée de $3 \mathrm{~kW}$.

En outre nous avons supposé que les paramètres du dispositif étudié avaient les valeurs suivantes :

$$
a=20 \mathrm{~mm}, d_{0}=200 \mathrm{~mm},
$$

soit $S=1 / 20$ ou encore $\sin \phi=a /\left(\sqrt{2} d_{0}\right) \approx 0,0707$ et $\phi \approx 4^{\circ}$.

Le caicul numérique a donc été réalisé à partir des deux expressions (10), (11).

Les coupes axiales de la répartition d'énergie dans le plan de superposition pour les trois valeurs du grandissement sont données sur la figure 2. Et la figure 3 donne la répartition d'énergie complète dans le plan de superposition pour un grandissement de 0,5 .

Afin de mettre en évidence l'influence du facteur de phase sur le résultat du calcul numérique nous avons calculé plus finement la répartition d'énergie au bord de l'image pour trois valeurs du paramètre $S$, à savoir : $S=1 / 15,1 / 20$ et $1 / 30$ en conservant toujours un grandissement $M=1$. Les résultats graphiques sont donnés sur la figure 4. Il apparaît qu'en général, on observe un maximum d'énergie au bord de la tache, et que si la condition (9) est satisfaite $(S=1 / 20)$, la période spatiale de la fluctuation énergétique a bien une largeur de $4 T$ conformément à ce qui avait été trouvé pour $\mathrm{L}_{1}$ [1]. C'est-àdire qu'elle est égale à environ $0,2 \mathrm{~mm}$ dans le cas présent (avec $\lambda=10,6 \mu \mathrm{m}, d_{\mathrm{i}}=200 \mathrm{~mm} \quad$ et $a=20 \mathrm{~mm}$ ).

Lorsque l'on s'écarte de cette condition, le profil de la fluctuation énergétique est tout de même pas mal modifié. Il paraît donc possible, selon l'applica- 


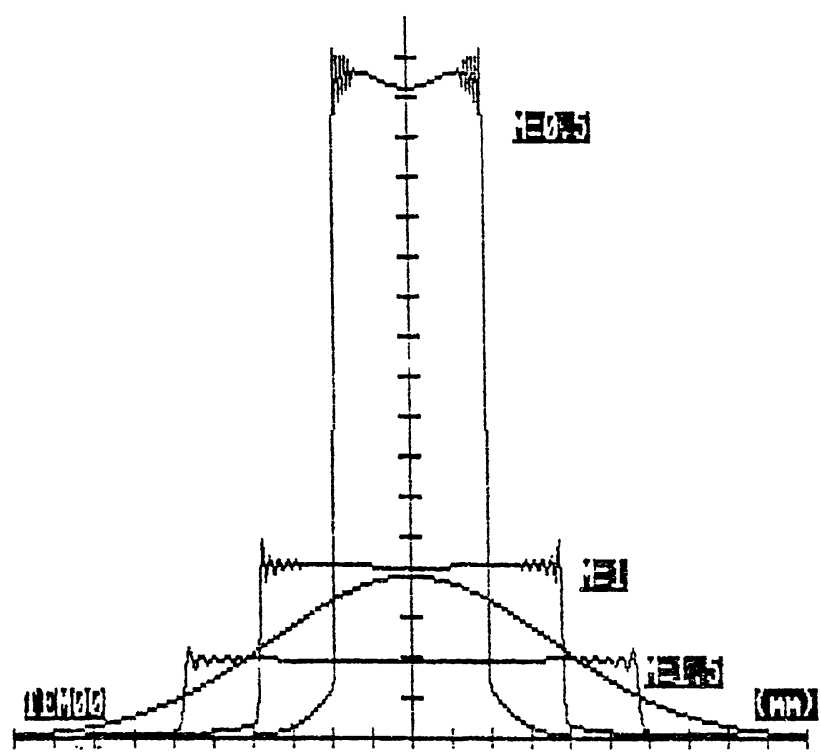

Fig. 2. - Coupes axiales de la répartition d'énergie dans le plan de superposition pour trois valeurs du grandissement $M=0,5-1$ et 1,5 ainsi que la coupe axiale du faisceau laser primaire.

[Axial section of energy distribution in the recombination plane for three values of optical magnification $M: 0.5-1$ 1.5 and axial section of primary laser beam.]

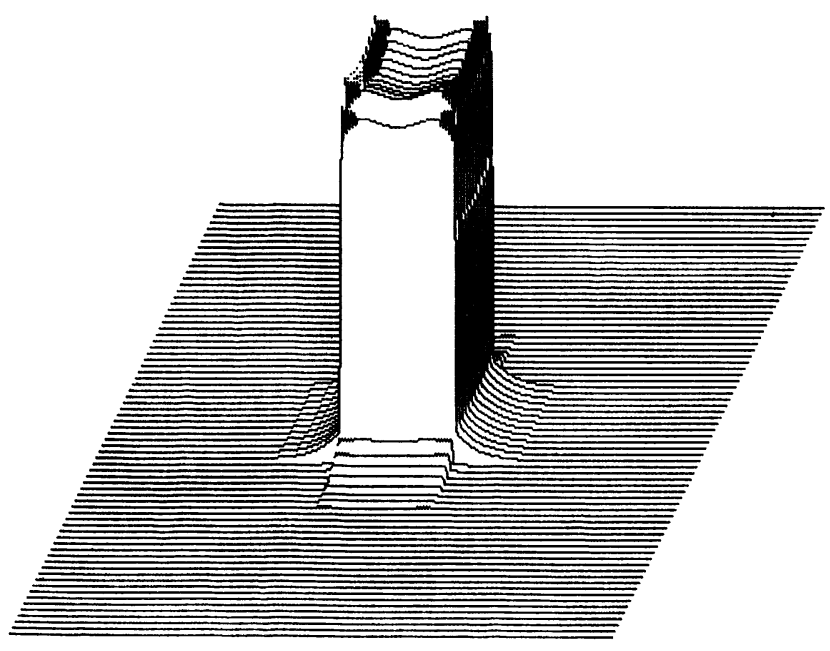

Fig. 3. - Répartition d'énergie complète dans le plan de superposition pour un grandissement de 0,5.

[Whole energy distribution in the superposition plane for $M=0.5$.]

tion envisagée, d'optimiser de cette façon la qualité de l'image. Cependant, ces fluctuations restant limitées à une zone étroite (quelques $T$, avec $T \approx 0,05 \mathrm{~mm}$ ) en bordure de la tache, cette amélioration n'est peut-être pas très importante pour le traitement thermique superficiel des matériaux métalliques. De toute façon cela n'est certainement

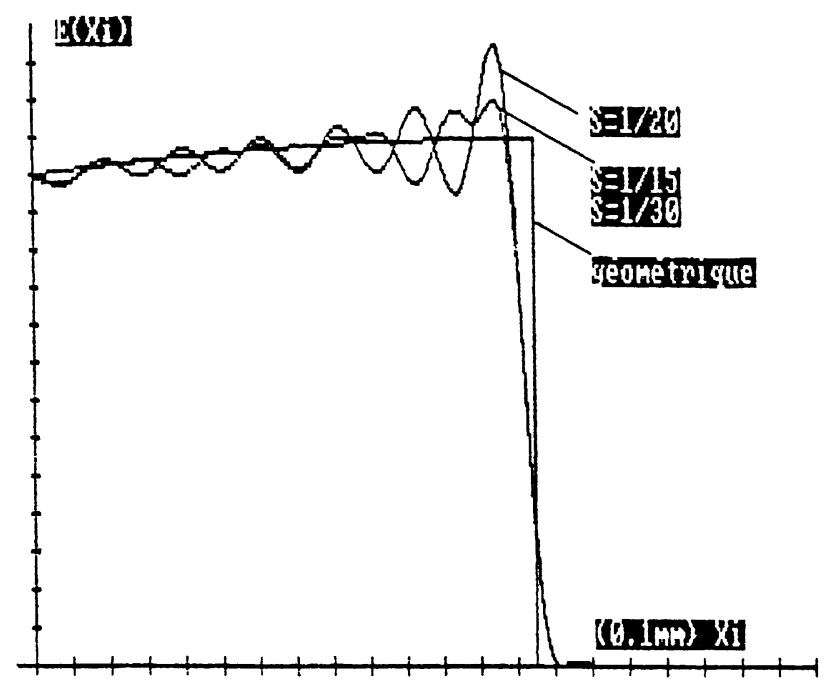

Fig. 4. - Coupes axiales de la répartition d'énergie au bord de l'image pour un grandissement $M=1$ et pour trois valeurs du paramètre $S: 1 / 15,1 / 20$ et $1 / 30$ ainsi que celle correspondant à l'optique géométrique.

[Axial section of energy distribution obtained at the edge of the image for $M=1$ and three values of $S$ parameter : $1 / 15,1 / 20,1 / 30$ and axial section deduced from geometrical optics.]

pas préjudiciable dans nombre d'applications, d'autant qu'un maximum énergétique en bordure de tache devrait compenser l'écoulement latéral de chaleur et donc conduire à un traitement globalement plus homogène, dès que l'on aura tendance à s'écarter du régime thermique transitoire [5].

\section{Discussion des influences dues aux tolérances pratiques.}

Les calculs précédents nous ont montré que le dispositif proposé peut condenser un faisceau laser gaussien en une tache carrée et homogène avec des dimensions variables. Toutefois, il faut noter que tout le calcul est basé sur les hypothèses suivantes : le faisceau laser primaire a une répartition d'énergie gaussienne, son axe correspond bien à celui du système optique et le plan de superposition idéal est confondu avec la surface du matériau à traiter. En toute rigueur, ces hypothèses sont souvent difficiles à satisfaire et la discussion concernant les influences liées aux écarts à ces situations idéales est intéressante à faire.

Bien que l'utilisation de la théorie scalaire de la diffraction permette d'analyser très précisément chaque situation, cela nécessiterait de nombreux calculs compliqués. Donc, pour simplifier, on se limitera ici à une analyse des deux situations les plus fréquemment rencontrées à savoir : l'influence du décalage entre l'axe optique du dispositif et celui du faisceau laser incident primaire ainsi que l'étude des déforma- 
tions de la répartition énergétique résultant d'une incertitude sur la position de la surface à traiter. Pour cela on utilisera la théorie de l'optique géométrique et on supposera encore que le faisceau laser incident primaire a une répartition d'énergie gaussienne.

\subsection{INFLUENCE D'UN DÉCALAGE ENTRE LES AXES} OPTIQUES DU DISPOSITIF ET DU FAISCEAU LASER PRIMAIRE. - Supposons que la position pratique du centre du faisceau primaire dans le plan d'entrée (plan qui passe par le sommet du miroir $\mathbf{M}_{1}$ et qui est perpendiculaire à l'axe du dispositif), soit située au point $(\Delta X, \Delta Y)$. En utilisant les coordonnées précédentes, la répartition d'énergie de l'image virtuelle s'exprime alors (pour simplifier, nous ne présenterons que les répartitions internes de la tache):

$$
\begin{aligned}
E_{0}\left(X_{0}, Y_{0}\right)=\operatorname{rect} & \left(X_{0} / 2 L\right) \operatorname{rect}\left(Y_{0} / 2 L\right) \times \\
& \times\left[\exp \left[-2\left(X_{0}-\Delta X-L\right)^{2} / w^{2}\right]\right. \\
& \left.+\exp \left[-2\left(X_{0}-\Delta X+L\right)^{2} / w^{2}\right]\right] \\
& \times\left[\exp \left[-2\left(Y_{0}-\Delta Y-L\right)^{2} / w^{2}\right]\right. \\
& \left.+\exp \left[-2\left(Y_{0}-\Delta Y+L\right)^{2} / w^{2}\right]\right]
\end{aligned}
$$

Selon l'approximation de l'optique géométrique, on obtient la répartition d'énergie de l'image réelle formée par la lentille $\mathrm{L}_{\mathrm{t}}$

$$
\begin{aligned}
E_{\mathrm{i}}\left(X_{\mathrm{i}}, Y_{\mathrm{i}}\right)= & \left(1 / M^{2}\right) E_{0}\left(-X_{\mathrm{i}} / M,-Y_{\mathrm{i}} / M\right) \\
& =\left(1 / M^{2}\right) \operatorname{rect}\left(X_{\mathrm{i}} / 2 M L\right) \times \\
& \times \operatorname{rect}\left(Y_{\mathrm{i}} / 2 M L\right) \\
& \times\left[\exp \left[-2\left(X_{\mathrm{i}}+M \Delta X+M L\right)^{2} / M^{2} w^{2}\right]\right. \\
& \left.+\exp \left[-2\left(X_{\mathrm{i}}+M \Delta X-M L\right)^{2} / M^{2} w^{2}\right]\right] \\
& \times\left[\exp \left[-2\left(Y_{\mathrm{i}}+M \Delta Y+M L\right)^{2} / M^{2} w^{2}\right]\right. \\
& \left.+\exp \left[-2\left(Y_{\mathrm{i}}+M \Delta Y-M L\right)^{2} / M^{2} w^{2}\right]\right]
\end{aligned}
$$

Il en résulte que ce décalage va diminuer la dimension de la zone «d'énergie homogène " dans la tache recomposée (Fig. 5). Pour bien évaluer la qualité de cette tache, on définira un facteur de qualité $C$ de la tache par l'expression :

$$
C=\left(E_{\max }-E_{\min }\right) /\left(E_{\max }+E_{\min }\right)
$$

où $E_{\max }$ et $E_{\min }$ sont respectivement les maxima et minima de la répartition d'énergie observés à l'intérieur de la tache.

Il est évident que plus ce facteur est petit plus la répartition d'énergie de la tache est homogène. En supposant que $|\Delta X|=|\Delta Y|$ et à partir des deux expressions (12), (13), la relation entre $C$ et le décalage $\Delta X$ (ou $\Delta Y$ ) est donnée par la figure 6 . Il en résulte que si $|\Delta X|$ et $|\Delta Y|$ sont inférieurs à $0,1 \mathrm{w}$, le facteur de qualité de la tache reste compris entre 0,03 et 0,08 . Dans ces conditions, la répartition d'énergie de la tache réelle pourra être considérée comme homogène.

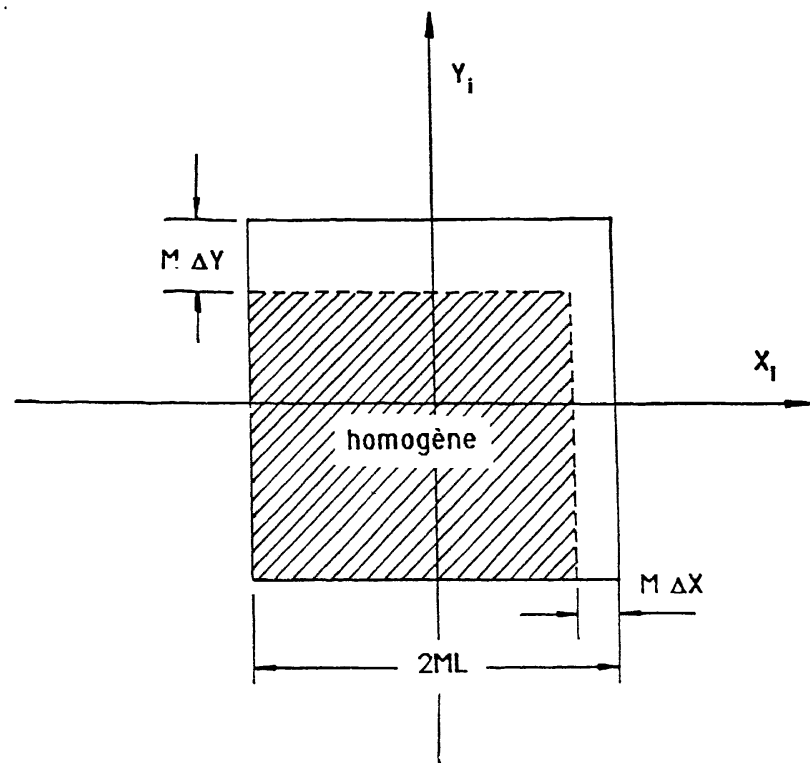

Fig. 5. - Influence d'un décalage entre l'axe optique du dispositif et le faisceau laser incident primaire.

[Effect of a gap between axe of optical device and primary laser beam.]

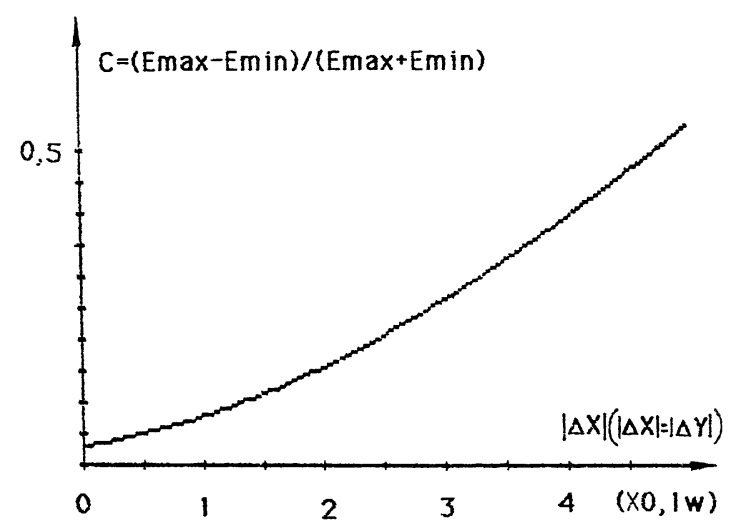

Fig. 6. - Influence sur le facteur de qualité d'un décalage entre les axes optiques du dispositif et du faisceau laser primaire.

[Gap effect on the quality factor.]

Toutefois, il faut noter d'après l'expression (12) que si un décalage n'est observé que dans une direction $(\Delta X$ ou $\Delta Y=0)$, la tache ne sera déformée que dans cette direction et que l'on pourra donc toujours l'utiliser pour réaliser un traitement superficiel homogène par balayage (à condition de choisir convenablement la direction de déplacement). Ce «défaut » peut d'ailleurs être mis à profit pour certaines applications en particulier pour avoir des traitements isothermes comme cela était montré dans [6]. Afin de mettre en évidence ce comportement, la figure 7 donne la forme de la répartition d'énergie lorsque $\Delta X=0,2 w$ et $\Delta Y=0$. 


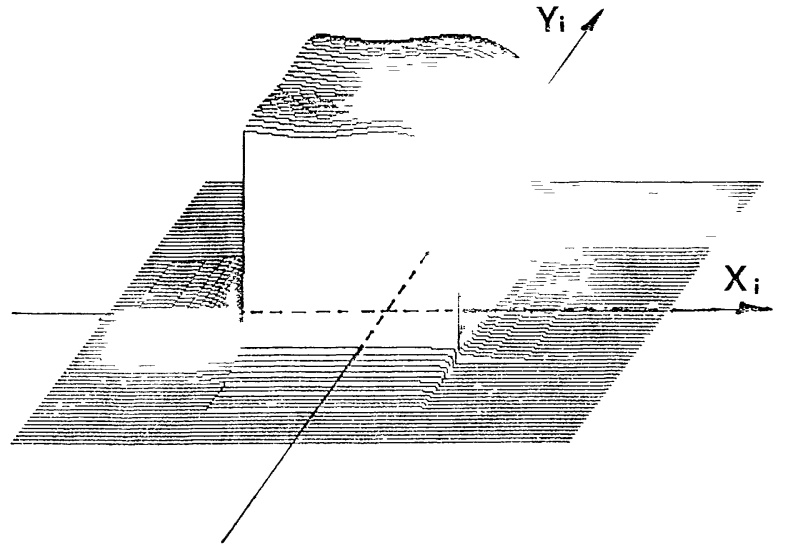

Fig. 7. - Répartition d'énergie correspondant à un décalage dans la seule direction de l'axe $X_{\mathrm{i}}$.

[Energy distribution consequent upon a gap only in the $X_{\mathrm{i}}$ axis direction.]

\subsection{INCERTITUDE SUR LA POSITION DE LA SURFACE} À TRAITER. - Supposons que la surface du matériau à traiter est située à une distance $d_{\mathrm{i}}+\Delta d_{\mathrm{i}}$ de la lentille $\mathrm{L}_{\mathrm{t}}$. Dans ce cas la loi de conjugaison des lentilles minces peut être exprimée par :

$$
\frac{1}{d_{0}+\Delta d_{0}}+\frac{1}{d_{\mathrm{i}}+\Delta d_{\mathrm{i}}}=\frac{1}{f}
$$

où $f$ est la distance focale de la lentille $\mathrm{L}_{\mathrm{t}}$.

Il est évident qu'on peut considérer la répartition de la tache au niveau de la surface à traiter comme celle de l'image réelle d'un « objet » virtuel situé à la distance $d_{0}+\Delta d_{0}$ en avant de la lentille $\mathrm{L}_{\mathrm{t}}$. Par conséquent, si $\Delta d_{\mathrm{i}}$ est suffisamment petit, on peut directement décrire la répartition d'énergie de l'image réelle par l'expression suivante :

$$
\begin{aligned}
E_{\mathrm{i}}^{\prime}\left(X_{\mathrm{i}}, Y_{\mathrm{i}}\right)= & \\
=\left(1 / M_{d}^{2}\right) & \operatorname{rect}\left(X_{\mathrm{i}} / 2 M_{d} L_{d}\right) \operatorname{rect}\left(Y_{\mathrm{i}} / 2 M_{d} L_{d}\right) \times \\
& \times\left[\exp \left[-2\left(X_{\mathrm{i}}-M_{d} L_{d}\right)^{2} / M_{d}^{2} w^{2}\right]\right. \\
& \left.+\exp \left[-2\left(X_{\mathrm{i}}+M_{d} L_{d}\right)^{2} / M_{d}^{2} w^{2}\right]\right] \\
& \times\left[\exp \left[-2\left(Y_{\mathrm{i}}-M_{d} L_{d}\right)^{2} / M_{d}^{2} w^{2}\right]\right. \\
& \left.+\exp \left[-2\left(Y_{\mathrm{i}}+M_{d} L_{d}\right)^{2} / M_{d}^{2} w^{2}\right]\right]
\end{aligned}
$$

avec

$$
M_{d}=\left(d_{\mathrm{i}}+\Delta d_{\mathrm{i}}\right) /\left(d_{0}+\Delta d_{0}\right)
$$

grandissement transversal pratique ;

$$
2 L_{d}=2 L+\sqrt{2} \cdot \Delta d_{0} \cdot \operatorname{tg} \phi,
$$

largeur pratique de «l'objet» virtuel.

Evidemment, une incertitude sur la position de la surface provoque non seulement une variation de la répartition d'énergie, mais aussi une variation des dimensions de la tache. D'après une simulation numérique, tant que $\Delta d_{\mathrm{i}}$ reste faible (de l'ordre du $\%)$ la répartition d'énergie de la tache obtenue reste assez homogène. A titre d'exemple la figure 8 présente la coupe axiale de la répartition d'énergie d'une tache pour les paramètres suivants : $w=7 \mathrm{~mm}, \quad 2 L=1, \quad 1 w, \quad d_{0}=200 \mathrm{~mm}, \quad d_{\mathrm{i}}=$ $200 \mathrm{~mm}, \phi=4^{\circ}$ et pour 2 positions de la surface correspondant à des écarts $\Delta d_{\mathrm{i}}= \pm 5 \mathrm{~mm}$, soit $\pm 2,5 \%$ par rapport à la position idéale $\left(\Delta d_{\mathrm{i}}=0\right)$. La figure 9 présente pour les mêmes paramètres l'influence de l'incertitude $\Delta d_{\mathrm{i}}$, sur le facteur de qualité $C$, sur la largeur pratique $2 L_{d}$ ainsi que sur la densité moyenne des taches réelles. Il apparaît très nettement que c'est surtout un rapprochement trop

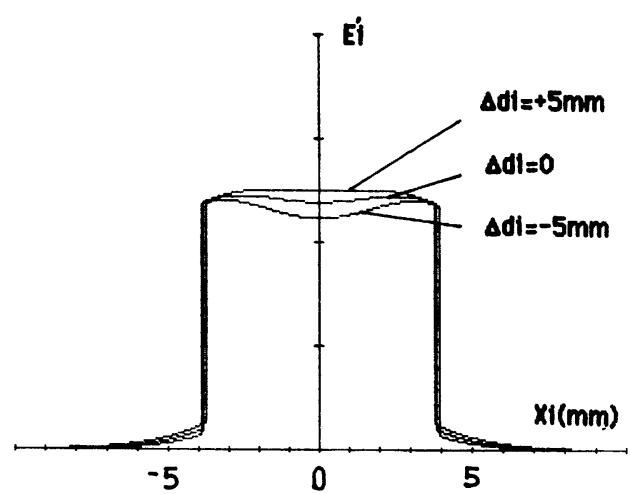

Fig. 8. - Coupes axiales des répartitions d'énergie de la tache selon la position de la surface à traiter par rapport au plan idéal de superposition.

[Axial section of energy distribution function of treated surface location in comparison with ideal position of superposition plane.]

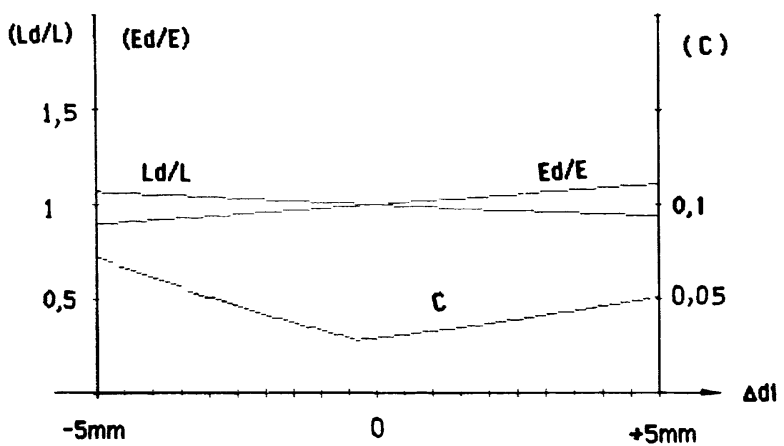

Fig. 9. - Influences de l'incertitude $\Delta d_{\mathrm{i}}$ de position du plan d'impact sur les caractéristiques de la répartition avec : $E_{d}$ : densité moyenne de la tache réelle ; $E$ : densité moyenne de la tache pour $\Delta d_{1}=0 ; 2 L_{d}$ : largeur de la tache réelle ; $2 L$ : largeur de la tache pour $\Delta d_{\mathrm{i}}=0 ; C$ : facteur de qualité.

[Effect of location incertitude $\Delta d_{\mathfrak{i}}$ on the distribution characteristics with : $E_{d}$ : mean density of actual spot ; $E$ : mean density of spot for $\Delta d_{\mathrm{i}}=0 ; 2 L_{d}$ : width of actual spot ; $2 L$ : width of spot for $\Delta d_{\mathrm{i}}=0 ; C$ : quality factor.] 
important de la position de la surface à traiter qui risque de poser problème vis-à-vis de l'homogénéité de la répartition d'énergie.

\section{Conclusion.}

Les analyses théoriques que nous venons de présenter montrent qu'à partir du dispositif décrit on peut facilement obtenir des taches carrées de dimensions variables et de bonne qualité. En outre, ce dispositif a une structure plus simple et plus compacte que celle du dispositif « $\mathrm{L}_{1}$ » précédemment étudié (voir Réf. [1]), ce qui devrait faciliter les problèmes de réglage optique pour les utilisateurs.
Bien entendu, les aberrations introduites par la lentille $\mathrm{L}_{t}$ n'ont pas été prises en compte, d'ailleurs dans la pratique il existe obligatoirement d'autres incertitudes, par exemple celles résultant de l'ajustement des pièces optiques ou encore de la répartition non gaussienne d'un faisceau laser réel. Cependant notre analyse théorique des modifications introduites par des écarts aux positions idéales laisse supposer que les qualités du dispositif ne sont pas notablement altérées tant que les incertitudes géométriques restent dans des tolérances raisonnables. Le présent dispositif devrait donc assez bien correspondre aux exigences des traitements thermiques superficiels.

\section{Bibliographie}

[1] Li J. C., Merlin J., Perez J., Revue de Phys. Appl. 21 (1986) 425-433.

[2] Li J. C., Merlin J., Perez J., Revue de Phys. Appl. 22 (1987) 201-204.

[3] Li Junchang, Journal of Optics (Paris), 18, $\mathrm{n}^{\circ} 2$ (1987).
[4] Goodman J. W., Introduction to Fourier Optics (Mac Graw-Hill Book Cy N.Y.) 1968.

[5] Dietz J., Merlin J., Revue de Phys. Appl. 23 (1988) 1787-1805.

[6] Merlin J., Dietz J., Revue de Phys. Appl. 23 (1988) 1807-1823. 